\title{
An Empirical Comparison of Transparency on One and Two Layer Displays
}

\section{Wael Aboelsaadat \& Ravin Balakrishnan}

\author{
Department of Computer Science, University of Toronto, \\ 10 King's College Road, Toronto, Ontario, Canada M5S $3 G 4$ \\ Email: \{wael,ravin\}@dgp.toronto.edu \\ URL: http://www.dgp.toronto.edu
}

Two layer displays are constructed by overlaying one transparent flat panel on another, with a discernible physical separation between layers. This layout could increase the available pixels without increasing the width and height of the display. However, it is unclear if the second physical layer provides any advantage over simple alpha-blended transparency on a single layer display. We investigate this issue in two controlled experiments that compare performance between one and two layer displays in users' perception of two potentially interfering virtual layers of information. Results show that for spatially overlapping stimuli, interference from the background stimuli on the perception of foreground stimuli is similar for both displays, while interference from the foreground stimuli on the perception of the background stimuli is higher with two layer displays. For spatially non-overlapping stimuli, perception is degraded on the two layer display if the distracter object is placed on the front layer.

Keywords: two layer displays, interference tasks, transparency.

\section{Introduction}

Recent technological advances and the demands for more screen real estate, sophisticated methods of interaction, and innovative visualization techniques have engendered a significant interest in non-traditional display designs. Two layer displays (e.g. http://www.deepvideo.com) have been proposed as an efficient alternative, since they can provide additional depth cues for use in 3D applications, and effectively double the available number of pixels with only a small ( $\sim$ inch) increase in the thickness of the display. Further, they are largely compatible with 
existing software and hardware systems, since they can be treated like a two monitor system, driven by a dual-head graphics card.

While these displays appear promising, without appropriate scientific knowledge about the effects of physically layered displays on user performance, interface designers cannot effectively utilize them. For example, one potential application would be to place user interface elements on one layer, and data on another. This notion of multiple layers of information has long been implemented using alpha-blending on a single layer display. Unlike two layer displays, alphablending on a single layer display does not increase the actual number of available pixels. In order to justify the cost of a second physical display layer, however, it is critical to understand if and how this setup improves upon alpha-blending on a single layer display apart from the simple increase in pixel count.

In this paper, we present empirical work which compares performance between one and two layer displays when interacting with two virtual layers of potentially interfering information. We explore several questions: Does physical separation change the amount of interference between foreground and background spatial stimuli? With two physical layers, can users better selectively process information on a specific layer and ignore the others? Will this change if the objects are spatially overlapping vs. if they are non-spatially overlapping? What is the effect of varying transparency across the layers? Does colour interact in a different way with interference level?

It is important to note that our experiment only addresses issues surrounding interference in the context of one layer vs. two layer displays. We do not address other possible benefits of two layer displays such as utilizing the physical separation to provide real depth in 3D applications (games, modelling, simulation, ... ). We also do not discuss using the two layer display in creating new information visualization techniques.

\section{Background}

There are three areas of research that are relevant to our work: attention, interference, and transparency.

\subsection{Attention}

The ability to direct user attention towards a specific object is a fundamental characteristic of any successful interface design. Several techniques have been proposed for guiding attention: spatial cues, alerts and graphical effects. Zhai et al. [1997] utilized masking to create bleaching, darkening, blurring, or screening effects to de-emphasize background material and thus causing the target to visually pop-up at the user. Harrison et al. [1995a] manipulated transparency to enhance performance in attentional tasks. Most of these techniques are based on colour change. However, perceptual psychologists have shown that depth is potentially more powerful than colour to help find an object [Nakayama \& Silverman 1986]. When the number of distracters in a colour-based task goes up, the search time goes up proportionally. On the other hand, when the number of distracters in a depth-based task goes up, the search time stays roughly constant [Triesman \& Gelade 1980]. 
It is unclear from the existing perceptual psychology literature whether users of a two layer display will be able to attend to single layer or not. Some researchers argue that attention cannot be preferentially allocated to specific locations in depth [Iavecchia \& Folk 1994; Ghiradelli \& Folk 1996; Theeuwes et al. 1998]. Hence, common depth is neither a necessary nor sufficient basis for attentional deployment. Others disagree and suggest that attention can be allocated to a specific location defined by disparity and that, when this is done, there is no interference from distracters in other depth planes [Nakayama \& Silverman 1986]. Other studies suggest that the deployment of attention across same-disparity loci is only possible when the elements being attended to are part of a well-formed surface with locally coplanar elements [He \& Nakayama 1995]. These studies show that it is difficult to attend to locations that span different surfaces. Further evidence for depth aware attention comes from studies that show that saccades to targets in different depth planes had longer saccadic latencies than saccades to targets in the same depth plane [Atchley et al. 1997]. Several models have also been proposed that attempt to reconcile attentional models in the third dimension. These attribute attentional deployment to the type of stimulus representation used in performing a given task. These reconciliatory models adopt a three level analysis: overall scene depth, layout of objects within the scene, and properties of objects [Andersen et al. 1998].

\subsection{Interference}

Often described as the index of attention by cognitive psychologists, the Stroop test provides insight into cognitive effects that are experienced as a result of interference [Stroop 1935]. The task takes advantage of our ability to read words more quickly and automatically than we can name colours. If a word is printed or displayed in a colour different from the colour it actually names; for example, if the word 'yellow' is written in blue ink, we will say the word 'yellow' more readily than we can name the colour in which it is displayed, which in this case is 'blue'.

In traditional Stroop tasks, a series of words are presented in randomly chosen colours. Participants must name the ink-colour while ignoring the word. Some of the words are neutral while other words are the names of conflicting colours. Consistent significant performance degradation occurs when conflicting colour words are used and participants attempt to name the colour of the ink. In other studies, a consistent and significant Stroop effect was found even when the word was printed in black ink, presented adjacent to a colour bar [Macleod 1991]. It is virtually impossible to consciously block or prevent the Stroop effect in selective looking tasks, despite numerous experimental permutations (over 700 articles — for reviews see Macleod [1991]). In our present work, we use variants of the Stroop test in evaluating possible differences between two layer displays and alpha-blending on a single layer display.

\subsection{Transparency}

Objects shown on the first layer of a two layered display are always transparent due to the physical properties of the constituent panels (Figure 1). Transparent interfaces, regardless of the number of physical layers in a display, have been proposed by several researchers to increase screen real estate and to provide interesting visualization and interaction techniques. Bier et al. [1993] proposed a new interface 


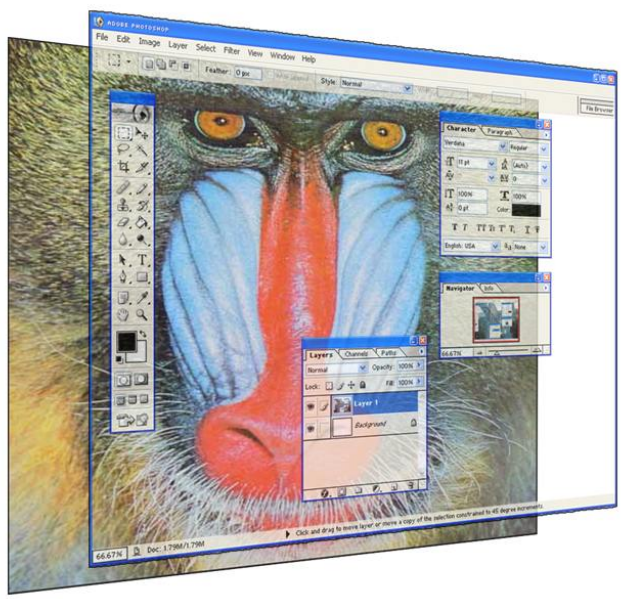

Figure 1: Two layer display. Front layer is transparent.

paradigm that used transparency to introduce a tight coupling between the tool function and the target object without occluding other interface objects. Harrison et al. [1995b] proposed using transparent user interfaces tools (menus, dialogue boxes, palettes, etc.) and information content windows. Ishii \& Kobayashi [1992] used transparency to overlay a drawing surface on a video image of the user's collaborative partner. Lieberman [1994] used multiple translucent layers to overlay zoomed-in and zoomed-out views of a scene. This prior art provides a significant basis for the use of transparent interfaces. However, it is unclear from the literature how a two layer display could change the efficacy of such interfaces compared to using alpha-blended transparency on a single layer display.

\section{Goal of the Current Work}

Our work is ultimately motivated by the desire to provide guidelines for the development of user interfaces for layered displays. The present work is one step in this direction, and our goal here is to obtain a better understanding of some of the factors that could affect users' ability to discriminate between foreground and background layers of information. We are primarily interested in how alpha-blending on a single layer display fares in comparison to a display with two physical layers. We conducted two experiments: the first investigated the situation where foreground and background stimuli were spatially overlapping, while the second investigated a non-spatially overlapping setup.

\section{Experiment 1: Spatially Overlapping Stimuli}

Our first experiment compares two layer to alpha-blended one layer displays with regards to users' ability to discriminate between foreground and background stimuli that are spatially overlapping. We use a variant of the Stroop test as our experimental 
task and vary several parameters, including the colour and content of the foreground and background information, level of transparency of the foreground stimulus, and content congruency between the foreground and background information.

\subsection{Apparatus}

We used a two layer display developed by Deep Video Imaging ${ }^{1}$, which has two panels: the front layer is physically transparent while the back layer is opaque (Figure 1). The display has a resolution of $1024 \times 768$ pixels on each layer, 24-bit colour depth, $170 \mathrm{Cd} \mathrm{m}^{-2}$ brightness, viewing angles of $140^{\circ}$ horizontal and $110^{\circ}$ vertical, and the separation between the two panels is $14.5 \mathrm{~mm}$. The display was driven by a $1 \mathrm{GHz}$ Pentium 3 computer running Windows2000. For the single layer display, we decided to use the back layer of the same two layer display to avoid introducing another variable into the experiment. An ANC650 (http://www.andreaelectronics.com) close-talk headset microphone with noise reduction facility was used as the input device. The microphone was connected to an Echo Mia high fidelity recording sound card (http://www.echoaudio.com). The experiments were run using Inquisit software (http://www.millisecond.com) and the voice recognition was done using the Microsoft speech recognition engine. Participants sat at a fixed distance of $750 \mathrm{~mm}$ from the screen.

\subsection{Participants}

Eighteen volunteers participated in the experiment. Two participants failed the pre-screening colour blindness test and were disqualified. The remaining sixteen (11 male, 5 female) passed the colour-blindness test, had normal or corrected-tonormal vision, and were naïve as to the purpose of the study. Participants were paid a nominal stipend for their participation, and could voluntarily withdraw without penalty at any time.

\subsection{Task and Stimuli}

The experiment had two tasks: a foreground focus (FF) task, and a background focus (BF) task. For both tasks, a coloured rectangle appeared in the foreground, and a black word appeared on the background in the same $x-y$ spatial location as the foreground stimulus (Figure 2). This is similar to the set-up used by Harrison et al. [1995a], except that we have two display conditions: in the two layer display condition, the foreground stimulus was displayed on the front display panel, and the background stimulus on the back display panel. For the single layer display condition, both stimuli were alpha-blended and displayed on the back, opaque, display panel of the same two layer display with the front panel left completely transparent.

\subsubsection{FF Task}

We used the colour-naming component of the Stoop test to measure how the perception of the foreground stimulus is affected by interference from the background stimulus. In this case, participants are asked to ignore the word in the background and name the colour of the rectangle in the foreground. By naming

\footnotetext{
${ }^{1}$ See http://www.deepvideo.com.
} 


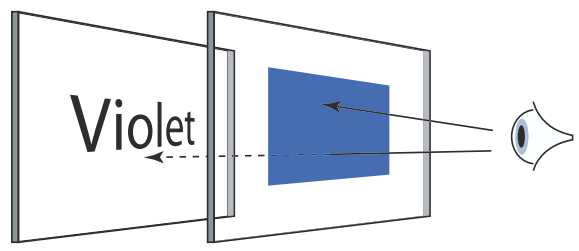

Figure 2: Experiment 1 setup (DUAL layer condition).

the colour and ignoring the word, the user, in effect, is performing a foreground information discrimination task in a spatially overlapping set-up. We will be measuring the time it takes the participant to name the colour, and error rates, on both display types. At high levels of transparency in the foreground stimulus (e.g. 100\% - clear), we anticipate that participants will experience high levels of interference from the word when they try to name the colour. As the foreground colour patch becomes more opaque, the interference from the word should decrease.

\subsubsection{BF Task}

We used the word-naming component of the Stoop test to measure how the perception of the background stimulus is affected by interference from the foreground stimulus. In this case, participants are asked to ignore the colour patch and read the word in the background. The colour patch in the foreground is always clearly visible and perceived. By reading the background word through the colour, the user is, in effect, performing a background information discrimination task in a spatially overlapping set-up. We will be measuring the time it takes the participants to read the word, and error rates, on both display types.

\subsection{Procedure}

At the start of the experiment, the task was explained to the participants. They were told to fixate on the centre of the display and either name the colour of the rectangle (in the FF task) or read the word (in the BF task). First, participants trained the speech recognition engine on how they pronounce the words and colour names. Next, participants were given a warm-up block of 15 unique trials randomly selected from the possible combinations, just to familiarize them with the task and conditions. Participants received feedback during the practice. If the answer was wrong, a 'WRONG!' message was displayed on screen. No error feedback was given during the experimental blocks. Once a participant provides response, the next trial is immediately displayed. Verbal responses were logged within $1 \mathrm{~ms}$ of accuracy. The experiment was conducted in one sitting and lasted about 120 minutes per participant. Participants were encouraged, through an on-screen display, to rest in the middle of each block for up to 3 minutes and at the end of each block for up to 15 minutes. At the end of the experiment, participants were debriefed and open-ended comments were recorded. 


\subsection{Hypotheses}

H1: Two layer display will enhance FF performance in a spatially overlapping layout.

H2: Two layer display will enhance BF performance in a spatially overlapping layout.

We anticipate more interference between foreground and background stimulus in the alpha-blended single layer display and therefore reduced performance in both $\mathrm{FF}$ and BF conditions.

\subsection{Design}

There were six experimentally manipulated conditions:

1. Rectangle Colour. Four colours were used: the three primary additive colours — Red, Green, Blue, and the fourth was a secondary additive colour: Yellow.

2. Word. Seven words (Helvetica, 34 point, uppercase) appeared through the colour rectangular patch. We used neutral words: UNCLE, NAIL, and CUTE in addition to the names of the four colours: RED, GREEN, BLUE and YELLOW.

3. Layer. Two set-ups were used. BACK - where the rectangle and word were displayed on a single layer using alpha-blending in the back panel. DUAL where the rectangle was displayed in the front panel and the word in the back panel.

4. Transparency. Seven transparency levels were used for the colour patch: 5\%, $7.5 \%, 10 \%, 25 \%, 50 \%, 75 \%$ and $100 \%$ (clear — both the word and colour show). The word naming experiment baseline condition was a word only presented with no colour rectangle showing. The colour naming experiment baseline condition was a colour only — presented with no word showing.

5. Task. Foreground focus - FF, and background focus - BF.

6. Stroop. Compound independent variable with 3 conditions: NEUTRAL - the word was a neutral word, INCONG - incongruent colour word was present, and CONG - colour word matched the colour of the patch.

A fully randomized, within participant, repeated measures design was used. The two task conditions and the two physical layer conditions were counter balanced between the participants: one group of eight participants did the FF condition first followed by BF condition, while the other group of eight did the BF condition followed by the FF condition. Within the first group, four participants did the BACK layer first and then the DUAL layer, while the other four participants did the DUAL layer first and then the BACK layer. The same treatment was applied to the second group of eight participants. Each participant was presented with three blocks, each consisting of all unique combinations appearing in random order within the block. 
The BF task had 406 unique trials calculated as follows:

7 words

$\times 4$ colours

$\times 7$ transparencies

$\times 2$ layers

+14 baseline cases ( 2 layers, 7 words)

$=406$ unique trial

The FF task had 400 unique trials calculated as follows:

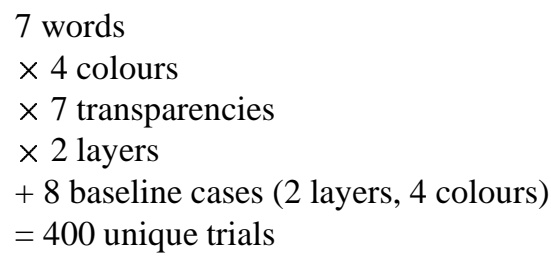

Hence, each participant did 2,418 trials. The total number of trials in the experiment is 38,688 .

\subsection{Results}

\subsubsection{FF Task}

Data from the warm-up trials was not used in our analysis. Participants' errors in response were very few $(\sim 2 \%)$ and these error trials were removed before subsequent data analysis. Outliers - calculated as trials with response times more than three std deviations from the mean - accounted for 5.2\% of the data, and were removed. A univariate repeated measures ANOVA was carried out on the remaining data. Significant main effects were found for layer $(F(1,47)=17.16, p<0.0001)$, confirming that two physical display layers affect ability to discern foreground stimulus layer differently from alpha-blending on a single display layer. Significant main effects were also found for transparency $(\mathrm{F}(6,282)=22.91, \mathrm{p}<0.0001)$ and Stroop $(\mathrm{F}(1,94)=379.36, \mathrm{p}<0.0001)$. This suggests that the Stroop effect was present and that transparency may indeed dilute the interference. Not surprisingly, colour also showed a significant main effect $(\mathrm{F}(3,141)=58.38, \mathrm{p}<0.0001)$ suggesting that saturation or luminance might dilute the interference.

Post-hoc analyses were carried out to compare means for physical layer. Despite the statistically significant difference, the overall mean for the DUAL condition was only $1.2 \%$ faster than the BACK condition. As expected, Stroop had three statistically significant groups: CONG, INCONG, and NEUTRAL. The CONG condition was 5\% faster than NEUTRAL, which in turn was $7.4 \%$ faster than INCONG. Analysis of variance showed a significant layer $\times$ Stroop interaction $(\mathrm{F}(5,235)=77.82, \mathrm{p}<0.0001)$. However, there was no significant difference between BACK-CONG and DUAL-CONG, BACK-INCONG and DUAL-INCONG, or BACK-NEUTRAL and DUAL-NEUTRAL pairs. Overall, this suggests that in the case where there is spatial overlap between foreground and background stimulus, in a task that requires focusing on the foreground stimulus, there is no difference 


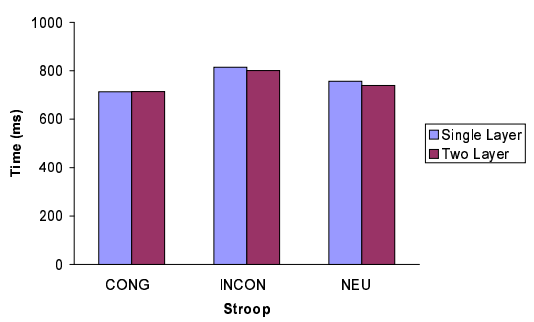

Figure 3: Mean response times for congruent, incongruent, and neutral conditions in FF task for all participants.

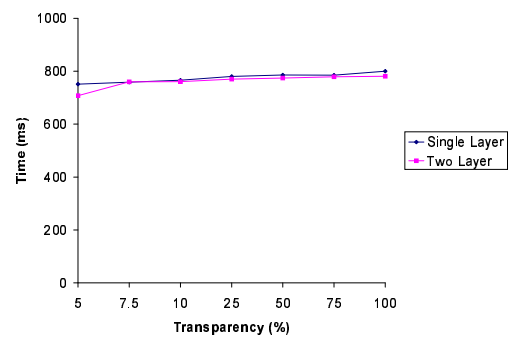

Figure 4: Mean response times for various transparency levels in the FF task for all participants.

of practical significance between single layer and dual layer displays. (Figure 3). Hence, Hypothesis $\mathrm{H} 1$ is rejected.

Post-hoc analyses were also carried out to compare means for the transparency levels. The means for transparency levels occurred in three statistically significant groupings: $100 \%+75 \%+50 \%+25 \%, 10 \%+7.5 \%$, and $5 \%$. Harrison et al. [1995a] have found similar groupings: $100 \%+50 \%+20 \%, 10 \%$ and $5 \%$. Analysis of variance showed a significant layer $\times$ transparency interaction $(\mathrm{F}(13,611)=10.18$, $\mathrm{p}<0.0001)$. The mean for $5 \%$ transparency was $6 \%$ faster in the two layer case suggesting that the physical depth decreased interference in this particular transparency level (Figure 4).

\subsubsection{BF Task}

Data from the warm-up trials was not used in our analysis. Participants' errors in response were very few $(\sim 2 \%)$ and these error trials were removed from subsequent data analysis. $3.9 \%$ of the data were identified as outliers in response time and also removed. A univariate repeated measures ANOVA was carried out on the remaining data. Significant main effects were found for layer $(F(1,47)=237.21, p<0.0001)$, 


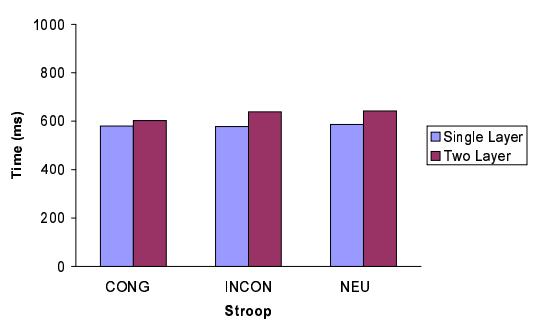

Figure 5: Response times for congruent, incongruent, and neutral conditions in the BF task for all participants.

transparency $(\mathrm{F}(6,282)=1040, \mathrm{p}<0.0001), \operatorname{colour}(\mathrm{F}(3,141)=309.16, \mathrm{p}<0.0001)$, and word $(F(5,282)=7.71, p<0.0001)$. This conforms with Harrison et al.'s [1995a] finding that legibility is affected by not only the level of transparency (visibility) but also the properties of the colour used (saturation and luminance). There was also an unexpected Stroop effect $(\mathrm{F}(1,94)=6.47, \mathrm{p}<0.0001)$.

Post-hoc analyses were carried out to compare means for layer. The mean for the DUAL condition was $8.6 \%$ slower than the BACK condition. Analysis of variance also showed a significant layer $\times$ Stroop interaction $(\mathrm{F}(5,235)=38.81$, $\mathrm{p}<0.0001)$. The mean for the INCONG-DUAL trials was $9.6 \%$ slower than INCONGBACK, and NEUTRAL-DUAL trials was $8.8 \%$ slower than NEUTRAL-BACK. There was no significant difference between CONG-DUAL and CONG-BACK. This indicates that in the case where there is spatial overlap between foreground and background stimulus, in a task that requires focusing on the background stimulus, two layers will perform $\sim 9.2 \%$ worse than a single layer (Figure 5). Hence, Hypothesis H2 is also rejected.

Post-hoc analyses were carried out to compare means for the transparency levels. The means for transparency levels occurred in five statistically significant groupings: $100 \%+75 \%+50 \%, 25 \%, 10 \%, 7.5 \%$ and $5 \%$. Analysis of variance showed a significant layer $\times$ transparency interaction $(\mathrm{F}(13,611)=355.63$, $\mathrm{p}<0.0001)$. The mean for DUAL-5\% trials is $14.1 \%$ slower than BACK-5\% trials, the mean for DUAL-7.5\% trials is $13.1 \%$ slower than BACK- $7.5 \%$ trials, and the mean for DUAL-10\% trials is $7.5 \%$ slower than BACK-10\% trials. This suggests that in the case where there are two spatially overlapping stimulus with the foreground stimulus being transparent by $10 \%$ or less, in a task that requires focusing on the background stimulus, two layers will perform on average $11 \%$ worse than a single layer (Figure 6).

Post-hoc analyses were also carried out to compare means for colour. The means for colour levels occurred in three statistically significant groupings: Blue, Red, and Green+Yellow. Analysis of variance showed a significant layer $\times$ colour interaction $(\mathrm{F}(7,329)=57.36, \mathrm{p}<0.0001)$. The mean for DUAL-Red was $7.9 \%$ 


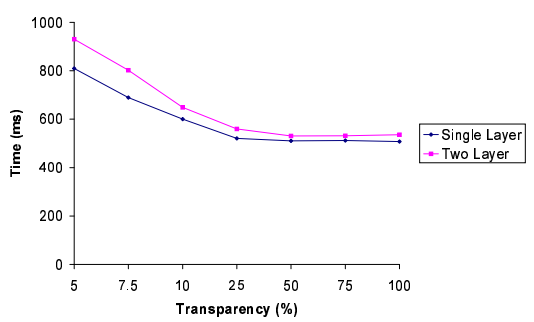

Figure 6: Mean response times for various transparency levels in the BF task for all participants.

slower than BACK-Red and the mean for DUAL-Blue was $11.7 \%$ slower than BACKBlue. Similarly, the mean for DUAL-Yellow was 5.5\% slower than BACK-Yellow, and the mean for DUAL-Green was $8.5 \%$ slower than BACK-Green. Hence, Yellow was the easiest to see through, followed by Red and Green, and finally Blue.

\section{Experiment 2: Spatially Non-Overlapping Stimuli}

In this experiment, stimuli are placed in proximity to each other but there is no spatial overlap between them. Our focus here is on the situation where potentially interfering elements are adjacent to the stimulus of interest, rather than overlapping as in the case of our first experiment.

\subsection{Apparatus}

The apparatus was the same as that in Experiment 1.

\subsection{Participants}

Twelve (8 male, 4 female) of the sixteen participants who participated in Experiment 1 were randomly selected for this experiment.

\subsection{Task and Stimuli}

We used the colour-naming component of the Stoop test to measure ability to focus on one portion of the display, while ignoring distracter stimulus on either the same or different layers of the display. In this case, a coloured line, 2 pixels thick, was displayed beneath a black word in the centre of the display (Figure 7). Participants were asked to ignore the word and name the colour of the line. By naming the colour and ignoring the word, the user, in effect, has to ignore the interference stimulus in a non-spatially-overlapping set-up. We will measure the time it takes to name the colour.

\subsection{Procedure}

A similar procedure to Experiment 1 was followed. The experiment was conducted in one sitting and lasted about 40 minutes per participant. 


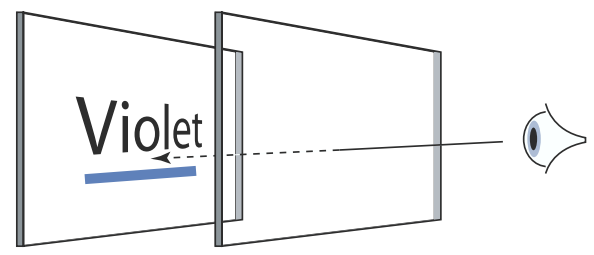

Figure 7: Experiment 2 setup (TextBack_LineBack condition).

\subsection{Hypotheses}

H3: Two layer display will enhance performance in a non-spatially overlapping layout.

\subsection{Design}

There were four experimentally manipulated conditions:

1. Line Colour. Six colours were used; the three primary additive colours: Red, Green and Blue, and three secondary additive colours: Yellow, Magenta and Cyan.

2. Word. Ten words (Helvetica, 34 point, uppercase) appeared above the coloured line. We used four neutral words: UNCLE, NAIL, FOOD and CUTE in addition to the six colour names: RED, GREEN, BLUE, YELLOW, MAGENTA and CYAN.

3. Layer. Three layouts were used: word in the back layer with line in the front layer (TextBack_LineFront), word in the front layer with line in the back layer (TextFront_LineBack), and word in the back layer with line in the back layer (TextBack_LineBack).

4. Stroop. Compound independent variable with 3 conditions: NEUTRAL - the word was a neutral word, INCONG - incongruent colour word was present, and CONG - colour word matched the colour of the line.

Participants were randomly divided into 6 groups of 2 each. Assignment of layer to groups was counterbalanced using a balanced Latin square. Each participant was presented with three blocks, each consisting of all unique combinations appearing in random order within the block. There were 198 unique trials as follows:

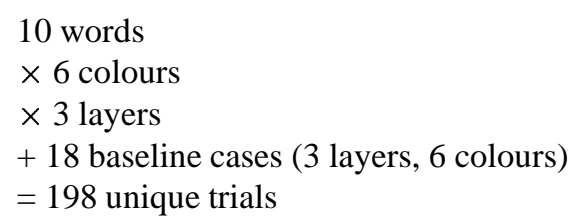

Hence, each participant did 594 trials. The total number of trials in the experiment is 7,128 . 


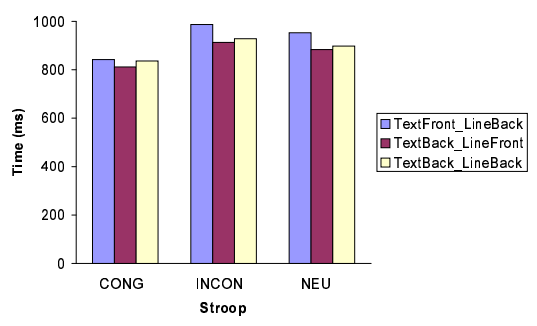

Figure 8: Response times for congruent, incongruent, and neutral conditions for all participants in Experiment 2.

\subsection{Results}

Data from the warm-up trials was not used in our analysis. Participants' errors in response were very few $(\sim 1 \%)$ and these error trials were removed from subsequent data analysis. $13.6 \%$ of the data were identified as outliers in response time and also removed. A univariate repeated measures ANOVA was carried out on the data. Significant main effects were found for layer $(F(2,70)=25.46, p<0.0001)$ and Stroop $(\mathrm{F}(1,70)=36.85, \mathrm{p}<0.0001)$. Not surprisingly, there was also a significant main effect for colour $(\mathrm{F}(5,175)=52.37, \mathrm{p}<0.0001)$, suggesting that saturation or luminance might dilute the interference even in a non-spatially overlapping layout.

Post-hoc analyses were carried out to compare means for layer. The means for layer levels occurred in two statistically significant groupings: TextFront_LineBack and TextBack_LineBack + TextBack_LineFront. The mean for TextFront_LineBack condition was $5.7 \%$ worse than the other two conditions. As expected, Stroop had three statistically significant groups: CONG, INCONG, and NEUTRAL. The CONG condition was $8.9 \%$ faster than NEUTRAL, which in turn was $4.1 \%$ faster than INCONG. Analysis of variance showed a significant layer $\times$ Stroop interaction $(\mathrm{F}(8,280)=12.69$, $\mathrm{p}<0.0001)$. The mean of TextFront_LineBack - INCONG trials was $7.5 \%$ worse than TextBack_LineBack - INCONG and TextBack_LineFront - INCONG. The mean of TextFront_LineBack - NEUTRAL trials was 5.5\% worse than TextBack_LineBack - NEUTRAL and TextBack_LineFront - NEUTRAL trials (Figure 8). There was no difference between the other combinations. This suggests that in the case where there are two spatially non-overlapping stimuli, a target and a distracter, two layers will always perform worse by an average of $6.5 \%$ if the distracter was placed in the front layer. Hence, Hypothesis H3 is rejected.

Post-hoc analyses were also carried out to compare means for colour. The means for colour levels occurred in four statistically significant groupings: Magenta, Cyan, Green+Blue+Red and Yellow. Analysis of variance showed a significant layer $\times$ colour interaction $(\mathrm{F}(17,595)=18.28, \mathrm{p}<0.0001)$. However, there was no significant difference for the same colour across layers. 


\section{Discussion and Conclusion}

We have presented experimental work that compared one and two layer displays with regards to users' ability to perceive two potentially interfering layers of information. Contrary to our initial hypotheses, the two layer display is not generally better than a single layer display. In a foreground focus task for spatially overlapping stimuli, both one and two layer displays perform similarly except at lower transparency levels where the two layer display performs better. In a background focus task for spatially overlapping objects, performance is dependent on the semantic relationship between the stimuli and also on transparency. In particular, performance of the two layer display degrades when the stimuli compete semantically for user attention. For nonspatially overlapping stimuli, performance is dependent on the assignment of stimuli to the various layers, with the single layer display equalling or outperforming the two layer display in all cases. While it is difficult to pinpoint the precise cause of the relatively poor overall performance of the two layer display in the interference tasks we studied, it is plausible that the physical separation of the two layers causes the human visual system to separate the viewed image into two constituent planes thus incurring additional processing cost. There may also be physical characteristics of the display that could be improved which could lead to performance improvements. However, given that our experiments were conducted using the same display for both the one and two layer conditions, it is unlikely that slight improvements in the (already very good) display quality would change the overall performance ranking. While our results are not terribly encouraging for two layer displays, it is important to note that our work was concerned only with issues surrounding interference between the layers. The other potentially significant benefit of two layer displays is the possibility of enhanced depth cues for $3 \mathrm{D}$ applications. Investigating this potential is left for future work.

\section{References}

Andersen, G., Braunstein, M. \& Saidpour, A. [1998], The Perception of Depth and Slant from Texture in Three-dimensional Scenes, Perception 27, 1087-106.

Atchley, P., Kramer, A., Anderesen, G. \& Theeuwes, J. [1997], Spatial Cuing in Stereoscopic Display: Evidence for a "Depth-aware" Attention Focus, Psychonomic Bulletin and Review 4, 525-9.

Bier, E. A., Stone, M. C., Pier, K., Buxton, W. \& DeRose, T. D. [1993], Toolglass and Magic Lenses: The See-through Interface, in J. Kajiya (ed.), Proceedings of SIGGRAPH'93 20th Annual Conference on Computer Graphics and Interactive Techniques, Computer Graphics (Annual Conference Series) 27, ACM Press, pp.73-80.

Ghiradelli, T. \& Folk, C. [1996], Spatial Cuing in a Stereoscopic Display: Evidence for a Depth Blind Attentional Spotlight, Psychonomic Bulletin and Review 3, 81-6.

Harrison, B., Ishii, H., Vicente, K. \& Buxton, W. [1995a], Transparent Layered User Interfaces: An Evaluation of a Display Design to Enhance Focused and Divided Attention, in I. Katz, R. Mack, L. Marks, M. B. Rosson \& J. Nielsen (eds.), Proceedings of the SIGCHI Conference on Human Factors in Computing Systems (CHI'95), ACM Press, pp.317-24. 
Harrison, B., Kurtenbach, G. \& Vicente, K. [1995b], An Experimental Evaluation of Transparent User Interface Tools and Information Content, in G. Robertson (ed.), Proceedings of the 8th Annual ACM Symposium on User Interface Software and Technology, UIST'95, ACM Press, pp.81-90.

He, Z. \& Nakayama, K. [1995], Visual Attention to Surfaces in Three-dimensional Space, Proceedings of the National Academy of Science-USA 92(24), 11155-9.

Iavecchia, H. \& Folk, C. [1994], Shifting Visual Attention in Stereographic Displays: A Time Course Analysis, Human Factors 36(4), 606-18.

Ishii, H. \& Kobayashi, M. [1992], ClearBoard: A Seamless Medium for Shared Drawing and Conversation With Eye Contact, in P. Bauersfeld, J. Bennett \& G. Lynch (eds.), Proceedings of the SIGCHI Conference on Human Factors in Computing Systems (CHI'92), ACM Press, pp.525-32.

Lieberman, H. [1994], Powers of Ten Thousand: Navigating in Large Information Spaces, in P. Szekely (ed.), Proceedings of the 7th Annual ACM Symposium on User Interface Software and Technology, UIST'94, ACM Press, pp.15-6.

Macleod, C. [1991], Half a Century of Research on the Stroop Effect: An Integrative Review, Psychological Bulletin 109(2), 163-203.

Nakayama, K. \& Silverman, G. [1986], Serial and Parallel Processing of Visual Features Conjunctions, Nature 320, 264-5.

Stroop, J. [1935], Studies of Interference in Serial Verbal Reactions, Journal of Experimental Psychology: General 18, 643-62.

Theeuwes, J., Kramer, A., Hahn, S. \& Irwin, D. [1998], Our Eyes Do Not Always Go Where We Want Them To Go: Capture Of The Eyes By New Objects, Psychological Science 5, 37985.

Triesman, A. \& Gelade, G. [1980], A Feature-integration Theory of Attention, Cognitive Psychology 12, 97-136.

Zhai, S., Wright, J., Selker, T. \& Klein, S. [1997], Graphical Means of Directing Users' Attention in the Visual Interface, in S. Howard, J. Hammond \& G. K. Lindgaard (eds.), Human-Computer Interaction - INTERACT '97: Proceedings of the Sixth IFIP Conference on Human-Computer Interaction, Chapman \& Hall, pp.59-66. 
Author Index

Aboelsaadat, Wael, 1

Balakrishnan, Ravin, 1 


\section{Keyword Index}

interference tasks, 1 transparency, 1

two layer displays, 1 
